Prons

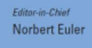
Journal of Nonlinear Mathematical Physics

\title{
Lax Integrability of the Modified Camassa-Holm Equation and the Concept of Peakons
}

Xiangke Chang, Jacek Szmigielski

To cite this article: Xiangke Chang, Jacek Szmigielski (2016) Lax Integrability of the Modified Camassa-Holm Equation and the Concept of Peakons, Journal of Nonlinear Mathematical Physics 23:4, 563-572, DOI:

https://doi.org/10.1080/14029251.2016.1248156

To link to this article: https://doi.org/10.1080/14029251.2016.1248156

Published online: 04 January 2021 


\title{
Lax Integrability of the Modified Camassa-Holm Equation and the Concept of Peakons
}

\author{
Xiangke Chang \\ LSEC, Institute of Computational Mathematics and Scientific Engineering Computing, AMSS, Chinese Academy of \\ Sciences, P.O.Box 2719, Beijing 100190, PR China \\ Department of Mathematics and Statistics, University of Saskatchewan, 106 Wiggins Road, Saskatoon, Saskatchewan, \\ S7N 5E6, Canada \\ changxk@lsec.cc.ac.cn \\ Jacek Szmigielski \\ Department of Mathematics and Statistics, University of Saskatchewan, 106 Wiggins Road, Saskatoon, Saskatchewan, \\ S7N 5E6, Canada \\ szmigiel@math.usask.ca
}

Received 17 August 2016

Accepted 2 September 2016

\begin{abstract}
In this Letter we propose that for Lax integrable nonlinear partial differential equations the natural concept of weak solutions is implied by the compatibility condition for the respective distributional Lax pairs. We illustrate our proposal by comparing two concepts of weak solutions of the modified Camassa-Holm equation pointing out that in the peakon sector (a family of non-smooth solitons) only one of them, namely the one obtained from the distributional compatibility condition, supports the time invariance of the Sobolev $H^{1}$ norm.
\end{abstract}

Keywords: Weak solutions; peakons; distributions.

2000 Mathematics Subject Classification: 35D30, 35Q51, 37J35, 35 Q53

\section{Introduction}

The partial differential equation with cubic nonlinearity

$$
\left.m_{t}+\left(\left(u^{2}-u_{x}^{2}\right) m\right)\right)_{x}=0, \quad m=u-u_{x x},
$$

is a modification of the Camassa-Holm equation (CH) $m_{t}+u m_{x}+2 u_{x} m=0, m=u-u_{x x}$ [6], for the shallow water waves. Originally, (1.1) appeared in the papers of Fokas [18], Fuchssteiner [21], Olver and Rosenau [45] and was, later, rediscovered by Qiao [46, 47]. According to the recent work [35] this equation has a number of potential applications and features that make this equation worth studying:

(1) it models the uni-directional propagation of shallow water waves over a flat bottom (in [35] the authors credit Fokas $[18,19]$ for the derivation);

(2) it arrises from an intrinsic (arc-length preserving) invariant planar flow in Euclidean geometry [26];

(3) it posses interesting non-smooth solutions such as non-smooth solitons (peakons). 
As to the name of (1.1) we note that the derivation of this equation in [45] followed from an elegant but mysterious method of tri-Hamiltonian duality applied to the bi-Hamiltonian representation of the modified Korteweg-de Vries equation. Since the $\mathrm{CH}$ equation can be obtained from the Korteweg-de Vries equation by the same tri-Hamiltonian duality, it is therefore natural to refer to (1.1) as the modified $\mathrm{CH}$ equation $(\mathrm{mCH})([26,40])$. To avoid confusions we point out that some authors use the name FORQ to denote (1.1) (e.g. [27, 28]). However, some ambiguity remains as there are other-distinct from (1.1)-equations also called the modified $\mathrm{CH}$ equation, obtained from the $\mathrm{CH}$ equation on the basis of other considerations (see e.g. [25]).

In the last 23 years since the appearance of [6] we have witnessed a sharp increase in reported "peakon equations": the first significant addition from this class was the Hunter-Saxton (HS) equation [3, 32,33], followed by the Degasperis-Procesi (DP) equation [15, 17], subsequently followed by V. Novikov equation [30,44] and then the Geng-Xue equation [23]. Around the same time, mainly through the work of Z.Qiao and his collaborators [46-49,51], with important contributions by others $[8,20,24,29,34,38]$, it became clear that the land of peakons is vast. This leads to a natural question of understanding and possibly classifying peakon equations using some, as yet unknown, fundamental principles.

It should be pointed out that the quest to understand the general principles behind the peakon equations is not motivated solely by purely mathematical interest. These equations show some remarkable features among which the stability of its peakon solutions is quite pertinent to this paper; we recall that the (orbital) stability of the $\mathrm{CH}$ peakons was established in [14], then the results for the periodic $\mathrm{CH}$ peakons [37] and the DP peakons [39] followed suit. Another reason for studying the peakon equations is their potential hydrodynamical significance [13], for example in capturing essential features of waves of greatest height $[9,10]$.

Before a classification of the peakon equations becomes viable it is important to make the following provisional statement: there are two types of known peakon equations, one type is in one way or the other described by Lax pairs, and equations in this class are expected to be integrable in some sense. The other type consists of non-integrable equations and the prototypical example was first introduced in the work of Degasperis, Holm and Hone [16] who defined a family of equations, later dubbed the $b$-equations,

$$
u_{t}-u_{x x t}+(b+1) u u_{x}=b u_{x} u_{x x}+u u_{x x x}, \quad b \in \mathbf{R},
$$

which reduce to $\mathrm{CH}$ and DP equations for special values $b=2, b=3$ respectively, while for other values of $b$ are non-integrable, failing the integrability test discussed in [17]; non-integrability later confirmed by more general methods by Mikhailov and Novikov in [43]. The main point of [16] was that regardless of the value of $b,(1.2)$ has peakon solutions obtained from the peakon ansatz $u=\sum_{j=1}^{n} m_{j}(t) e^{-\left|x-x_{j}(t)\right|}$, by constraining smooth coefficients $m_{j}(t), x_{j}(t)$ to satisfy a system of ODEs.

At this point our interest is in understanding the mathematical underpinnings of Lax integrable peakon equations, leaving non-integrable peakon equations for future work. In our opinion two features of (1.1) deserve to be further studied to inform future discussions of Lax integrable peakon equations:

(1) as we argue below, using (1.1) as an example, the definition of what one means by weak solutions of a Lax integrable peakon equation is determined by the distributional character of its Lax pair; 
(2) the Lax integrable peakon ODEs (see (2.12)) are isospectral deformations of an oscillatory system in the sense of Gantmacher and Krein [22].

It is fair to say that these two items have very different ontological status. The question as to what one means by weak solutions in the case of an obvious lack of sufficient classical smoothness is a necessary starting point for any serious discussion of a PDE to which one is seeking generalized (non classical) solutions. So without proper understanding of this issue it will be hard to have any meaningful discussion of what peakon equations are. By contrast, the fact that the peakon sector of the DP equation comes from an oscillatory system was first proven in [41], later confirmed for V. Novikow peakons in [31], for the Geng-Xue equation in [42], while for the $\mathrm{CH}$ equation it is implicit in $[1,2]$ once one realizes that the $\mathrm{CH}$ equation can be viewed as an isospectral deformation of the inhomogeneous string as defined by M. G. Krein in his study of oscillatory systems [22]. So the second item is just a confirmation that the mysterious link between peakons and oscillatory systems persists for (1.1) and we have no additional insight into this issue at the moment, so we restrict ourselves just to mentioning this fact. The remainder of the present letter is confined entirely to the first item on the list above.

\section{Weak solutions of the modified $\mathrm{CH}$ equation; a comparison}

We note that equation (1.1) is the first peakon equation known to us for which the need to view the weak sector as a consequence of the distributional compatibility of its Lax pair - rather than by defining weak solutions a priori, basing the definition on the type of nonlinearity - is placed front and center.

We recall that (1.1) can be written in bi-Hamiltonian form [45]

$$
m_{t}=\mathscr{K} \frac{\delta \mathscr{H}_{1}}{\delta m}=\mathscr{J} \frac{\delta \mathscr{H}_{2}}{\delta m}, \quad m=u-u_{x x},
$$

where the compatible Hamiltonian operators are:

$$
\mathscr{K}=-\partial_{x} m \partial_{x}^{-1} m \partial_{x}, \quad \mathscr{J}=\left(\partial_{x}^{3}-\partial_{x}\right),
$$

with Hamiltonian functionals

$$
\mathscr{H}_{1}=\int \operatorname{mudx}, \quad \mathscr{H}_{2}=\frac{1}{4} \int\left(u^{4}+2 u^{2} u_{x}^{2}-\frac{1}{3} u_{x}^{4}\right) d x .
$$

Even though these are formal expressions it is not difficult to make rigorous analytic sense of them. For example, if $u \in \mathscr{S}(\mathbf{R})$ (Schwartz class of smooth, rapidly vanishing functions) then all expressions are well defined, in particular $\partial_{x}^{-1}$ can be taken to act as $\int_{-\infty}^{x}$ on any function $f \in \mathscr{S}(\mathbf{R})$, giving after one integration by parts

$$
\mathscr{H}_{1}=\int_{\mathbf{R}}\left(u^{2}+u_{x}^{2}\right) d x=\|u\|_{H^{1}}^{2} .
$$

In other words the Hamiltonian $\mathscr{H}_{1}$ is the square of the Sobolev norm. Since Hamiltonians are constants of motion we see that at least in the smooth sector of solutions the Sobolev norm, $\|u\|_{H^{1}}$, is a constant of motion. The objective now is to extend (1.1) to functions with less smoothness, preserving as much of the original structure as possible. We recall from [26] one possible definition of weak solutions. 
Definition 2.1 (Weak solution). Given initial data $u_{0}$ in the Sobolev space $W^{1,3}(\mathbf{R})$, the function $u \in L_{\text {loc }}^{\infty}\left([0, T], W_{\text {loc }}^{1,3}(\mathbf{R})\right)$ is said to be a weak solution to (1.1) with initial condition $u_{0}$ if the following identity holds

$$
\int_{0}^{T} \int_{\mathbf{R}}\left[u \phi_{t}+\frac{1}{3} u^{3} \phi_{x}+\frac{1}{3} u_{x}^{3} \phi+p \star\left(\frac{2}{3} u^{3}+u u_{x}^{2}\right) \phi_{x}-\frac{1}{3}\left(p \star u_{x}^{3}\right) \phi\right] d x+\int_{\mathbf{R}} u_{0}(x) \phi(0, x) d x=0
$$

for all test functions $\phi(t, x) \in C_{c}^{\infty}([0, T) \times \mathbf{R})$, where $p(x)=\frac{1}{2} e^{-|x|}$ and the star $\star$ is the standard convolution product on $\mathbf{R}$ (for other details see [26]).

We will test this definition on an example of a non-smooth solution obtained from the peakon ansatz $[6,26,49]$, that is, we assume

$$
u=\sum_{j=1}^{n} m_{j}(t) e^{-\left|x-x_{j}(t)\right|}
$$

where all $m_{j}$ s are positive, and hence $m=2 \sum_{j=1}^{n} m_{j} \delta_{x_{j}}$ is a positive discrete measure. We then substitute this ansatz into Definition 2.1 to determine the $t$-dependence of $m_{j}(t), x_{j}(t)$. The relevant computation is done in [26] and we record the result following the elegant presentation in [49]:

$$
\dot{m}_{i}=0, \quad \dot{x}_{i}=-\frac{1}{3} m_{i}^{2}+\sum_{j, k}^{n} m_{j} m_{k}\left(1-\operatorname{sgn}\left(x_{i}-x_{j}\right) \operatorname{sgn}\left(x_{i}-x_{k}\right)\right) e^{-\left|x_{j}-x_{i}\right|-\left|x_{i}-x_{k}\right|},
$$

with the convention $\operatorname{sgn}(0)=0$. We can specialize this expression to the set satisfying the ordering conditions $x_{1}<x_{2}<\cdots<x_{n}$, in which case (2.7) simplifies to:

$$
\dot{m}_{j}=0, \quad \dot{x}_{j}=\frac{2}{3} m_{j}^{2}+2 m_{j} \sum_{i \neq j} m_{i} e^{-\left|x_{i}-x_{j}\right|}+4 \sum_{i<j<k}^{n} m_{i} m_{k} e^{-\left|x_{i}-x_{k}\right|} .
$$

Remark 2.1. We believe there is a slight misprint in [26] (see p. 22 therein), namely, the second term should have only non-diagonal summation as displayed above. We also note that stability of peakons satisfying (2.8) was established in [50] and [40].

It is sufficient for our purposes to assume the number of peakons $n$ to be two. Thus

$$
u=m_{1}(t) e^{-\left|x-x_{1}(t)\right|}+m_{2}(t) e^{-\left|x-x_{2}(t)\right|},
$$

and the above definition of a weak solution in this special case results in the following system of ODEs:

$$
\begin{aligned}
& \dot{m}_{1}=0=\dot{m}_{2}, \\
& \dot{x}_{1}=\frac{2}{3} m_{1}^{2}+2 m_{1} m_{2} e^{-\left|x_{1}-x_{2}\right|}, \\
& \dot{x}_{2}=\frac{2}{3} m_{2}^{2}+2 m_{1} m_{2} e^{-\left|x_{1}-x_{2}\right|} .
\end{aligned}
$$

The Sobolev norm of $u$ for the two-peakon ansatz can be easily computed (see the first part of the proof of Theorem 2.1 for the general case) to be

$$
\begin{gathered}
\|u\|_{H^{1}}^{2}=2 m_{1} u\left(x_{1}\right)+2 m_{2} u\left(x_{2}\right)=2\left(m_{1}^{2}+m_{2}^{2}\right)+4 m_{1} m_{2} e^{-\left|x_{1}-x_{2}\right|}, \\
\text { Co-published by Atlantis Press and Taylor \& Francis } \\
\text { Copyright: the authors }
\end{gathered}
$$


and this expression is clearly not $t$ invariant for general $m_{1}, m_{2}$ as one can easily see by computing $\frac{d}{d t}\|u\|_{H^{1}}^{2}$ in the region $x_{1}<x_{2}$ with the help of (2.9), in which case one obtains

$$
\frac{d}{d t}\|u\|_{H^{1}}^{2}=4 m_{1} m_{2}\left(\dot{x}_{1}-\dot{x}_{2}\right) e^{x_{1}-x_{2}}=\frac{8 m_{1} m_{2}}{3}\left(m_{1}^{2}-m_{2}^{2}\right) e^{x_{1}-x_{2}},
$$

implying that the Sobolev $H^{1}$ norm of $u$ is only conserved in the case of $m_{1}=m_{2}$.

Remark 2.2. The concept of a weak solution proposed in [26] is a natural generalization of earlier definitions of weak solutions developed for nonlinear PDEs of Burgers' type, in other words inspired by one dimensional conservation laws (see $[11,12]$ for a relevant discussion of this type of weak solutions to $\mathrm{CH}$; for other types of weak solutions to $\mathrm{CH}$ see [5]) and it works well for certain fundamental questions, like general existence theorems. We are only pointing out that, perhaps essential, aspects of integrability known in the smooth sector may not survive the transition to the weak sector so defined. For other relevant work related to (1.1) and its weak solutions the reader is asked to consult [4].

\subsection{Lax integrability and peakons}

What we are proposing in this paper is to preserve Lax integrability instead and let Lax integrability dictate the suitable paradigm for weak/distributional solutions of (1.1). First we state the final result in the case of the peakon ansatz, leaving the details to the later part of the paper. In order to view (1.1) as a distributional equation involving a discrete measure $m$ one needs to define the product $u_{x}^{2} m$. We will argue below that the only choice consistent with Lax integrability is to take $u_{x}^{2} m$ to mean

$$
u_{x}^{2} m \stackrel{\text { def }}{=}\left\langle u_{x}^{2}\right\rangle m
$$

where $\langle f\rangle$ denotes the average function (the arithmetic average of the right hand and left hand limits). Since for the peakon ansatz

$$
\dot{m}=2 \sum_{j=1}^{n} \dot{m}_{j} \delta_{x_{j}}-2 \sum_{j=1}^{n} m_{j} \dot{x}_{j} \delta_{x_{j}}^{\prime}
$$

hence (1.1), with the rule (2.10) in force, readily reduces to the system of ODEs:

$$
\dot{m}_{j}=0, \quad \dot{x}_{j}=u^{2}\left(x_{j}\right)-\left\langle u_{x}^{2}\right\rangle\left(x_{j}\right) .
$$

Furthermore, assuming the ordering condition $x_{1}<x_{2}<\cdots<x_{n}$, we obtain another, more explicit form of (2.12), namely

$$
\dot{m}_{j}=0, \quad \dot{x}_{j}=2 \sum_{\substack{1 \leq k \leq n, k \neq j}} m_{j} m_{k} e^{-\left|x_{j}-x_{k}\right|}+4 \sum_{1 \leq i<j<k \leq n} m_{i} m_{k} e^{-\left|x_{i}-x_{k}\right|} .
$$

We note that this system differs from (2.8); the difference amounting to the absence of the term $\frac{2}{3} m_{j}^{2}$ whose presence on the other hand can be traced back precisely to the definition of the singular product $u_{x}^{2} m$. Indeed, if we used Definition 2.1 then

$$
u_{x}^{2} m \stackrel{\operatorname{def} 2.1}{=}\left(\frac{\left\langle u_{x}^{2}\right\rangle+2\left\langle u_{x}\right\rangle^{2}}{3}\right) m
$$




\subsection{Lax integrability and the preservation of the $H^{1}$ norm}

The following theorem supports the idea of building the concept of weak solutions to (1.1) based on the multiplication formula (2.10). We have

Theorem 2.1. Let $u$ be given by the peakon ansatz (2.6) and let the multiplication of the singular term $u_{x}^{2} m$ in (1.1) be defined by (2.10). Then if $u$ satisfies (1.1) and

$$
\frac{d}{d t}\|u\|_{H^{1}}=0
$$

Proof. First we establish that for the peakon ansatz the relation (see (2.4))

$$
\|u\|_{H^{1}}^{2}=\int u m d x=\sum_{j=1}^{n} 2 m_{j} u\left(x_{j}\right)
$$

persists. Indeed, setting $x_{0}=-\infty, x_{n+1}=\infty$ and partitioning $\mathbf{R}$ as $\cup_{j=0}^{n}\left(x_{j}, x_{j+1}\right)$, we can write

$$
\begin{aligned}
& \|u\|_{H^{1}}^{2}=\int\left(u^{2}+u_{x}^{2}\right) d x=\sum_{j=0}^{n} \int_{x_{j}+}^{x_{j+1}-}\left(u^{2}+u_{x}^{2}\right) d x= \\
& \int u^{2} d x+\left.\sum_{j=0}^{n} u u_{x}\right|_{x_{j}+} ^{x_{j+1}-}-\sum_{j=0}^{n} \int_{x_{j}+}^{x_{j+1}-} u u_{x x} d x=\left.\sum_{j=0}^{n} u u_{x}\right|_{x_{j}+} ^{x_{j+1}-},
\end{aligned}
$$

where in the last step we used that $u=u_{x x}$ holds away from the support of $m$. If we denote by $[f]\left(x_{j}\right)$ the jump of a piecewise continuous function $f$ at $x_{j}$ then we can write

$$
\|u\|_{H^{1}}^{2}=-\sum_{j=1}^{n}\left[u_{x}\right]\left(x_{j}\right) u\left(x_{j}\right)=\sum_{j=1}^{n} 2 m_{j} u\left(x_{j}\right)=\int u m d x
$$

thus proving the claim. We proceed now to compute the time derivative of $\|u\|_{H^{1}}^{2}$. We will carry out the computation in two steps. First we observe that as long as all $x_{j} \mathrm{~s}$ are distinct, $u\left(x_{j}\right)$ is differentiable in $t$ and the derivative is given by the formula

$$
\frac{d}{d t} u\left(x_{j}\right)=\left\langle u_{x}\right\rangle\left(x_{j}\right) \dot{x}_{j}
$$

which in conjunction with (2.10) and (2.13) implies

$$
\begin{aligned}
& \frac{d}{d t}\|u\|_{H^{1}}^{2}=\frac{d}{d t}\left(\sum_{j=1}^{n} 2 m_{j} u\left(x_{j}\right)\right)=\sum_{j=1}^{n} 2 m_{j}\left\langle u_{x}\right\rangle\left(x_{j}\right) \dot{x}_{j} \stackrel{(2.12)}{=} \sum_{j=1}^{n} 2 m_{j}\left\langle u_{x}\right\rangle\left(x_{j}\right)\left(u^{2}-\left\langle u_{x}^{2}\right\rangle\right)\left(x_{j}\right)= \\
& -\sum_{j=1}^{n}\left[u_{x}\right]\left(x_{j}\right)\left\langle u_{x}\right\rangle\left(x_{j}\right)\left(u^{2}-\left\langle u_{x}^{2}\right\rangle\right)\left(x_{j}\right)=-\frac{1}{2} \sum_{j=1}^{n}\left[u_{x}^{2}\right]\left(x_{j}\right)\left(u^{2}-\left\langle u_{x}^{2}\right\rangle\right)\left(x_{j}\right)= \\
& \frac{1}{2} \sum_{j=1}^{n}\left[u^{2}-u_{x}^{2}\right]\left(x_{j}\right)\left\langle u^{2}-u_{x}^{2}\right\rangle\left(x_{j}\right)=\frac{1}{4} \sum_{j=1}^{n}\left[\left(u^{2}-u_{x}^{2}\right)^{2}\right]\left(x_{j}\right),
\end{aligned}
$$

where we used multiple times the identity $\langle f\rangle\left(x_{j}\right)[f]\left(x_{j}\right)=\frac{1}{2}\left[f^{2}\right]\left(x_{j}\right)$ valid for any piecewise continuous function $f$. This establishes the identity

$$
\frac{d}{d t}\|u\|_{H^{1}}^{2}=\frac{1}{4} \sum_{j=1}^{n}\left[\left(u^{2}-u_{x}^{2}\right)^{2}\right]\left(x_{j}\right)
$$


which completes the first step of the computation. In the second step of the computation we note that for the peakon ansatz (2.6) $u^{2}-u_{x}^{2}$ is a piecewise constant function since on each interval $\left(x_{j}, x_{j+1}\right)$

$$
u=A_{j} e^{x}+B_{j} e^{-x}, \quad u_{x}=A_{j} e^{x}-B_{j} e^{-x},
$$

hence $u^{2}-u_{x}^{2}=4 A_{j} B_{j}$ there. Moreover, on the interval $\left(x_{0}, x_{1}\right), B_{0}=0$, and on $\left(x_{n}, x_{n+1}\right), A_{n}=0$ respectively, implying in each case that $u^{2}-u_{x}^{2}=0$ on these two intervals. In the final step of the proof we use that $u^{2}-u_{x}^{2}$ is a piecewise constant function to note that $\sum_{j=1}^{n}\left[\left(u^{2}-u_{x}^{2}\right)^{2}\right]\left(x_{j}\right)$ is therefore a telescoping sum and hence, as the result of cancellations of interior terms and the absence of boundary terms,

$$
\frac{d}{d t}\|u\|_{H^{1}}^{2}=\frac{1}{4}\left(\left(u^{2}-u_{x}^{2}\right)^{2}\left(x_{n}+\right)-\left(u^{2}-u_{x}^{2}\right)^{2}\left(x_{1}-\right)\right)=0,
$$

which completes the proof.

\section{Lax integrability and weak solutions}

Here we provide some details as to why the originally ill-defined term $u_{x}^{2} m$ in (1.1) should be regularized by using formula (2.10) if Lax integrability is to be preserved. We first recall that in the smooth sector the Lax pair for (1.1) reads [46]:

$$
\Psi_{x}=\frac{1}{2} U \Psi, \quad \Psi_{t}=\frac{1}{2} V \Psi, \quad \Psi=\left[\begin{array}{l}
\Psi_{1} \\
\Psi_{2}
\end{array}\right]
$$

with

$$
U=\left[\begin{array}{cc}
-1 & \lambda m \\
-\lambda m & 1
\end{array}\right], \quad V=\left[\begin{array}{cc}
4 \lambda^{-2}+Q & -2 \lambda^{-1}\left(u-u_{x}\right)-\lambda m Q \\
2 \lambda-2\left(u+u_{x}\right)+\lambda m Q & -Q
\end{array}\right], \quad Q=u^{2}-u_{x}^{2} .
$$

Furthermore, in the smooth sector, Lax integrability is understood to mean that the compatibility condition $\Psi_{x t}=\Psi_{t x}$ implies (1.1). To promote (3.1) to a distributional Lax pair we need to ensure that all sides of (3.1) are well defined as distributions. In our approach we consider $\Psi(\bullet, t)$ as a vector valued $\left(\mathbf{R}^{2}\right)$ distribution in $\mathscr{D}^{\prime}\left(\mathbf{R}_{x}\right)$ and $D_{x}$ is the standard distributional derivative (in $\left.x\right)$. We view the $t$ variable as a deformation parameter, in agreement with the original formulation by $\mathrm{P}$. Lax [36], which prompts us to view $\Psi(\bullet, t)$ as a differentiable map.

Definition 3.1. Let $T>0$ be given then $\Psi(\bullet, t)$ is a differentiable map

$$
\Psi(\bullet, t):(0, T) \ni t \longrightarrow \mathbf{R}^{2} \otimes \mathscr{D}^{\prime}\left(\mathbf{R}_{x}\right),
$$

where the distributional derivative (with respect to $t$ ) is given by

$$
D_{t} \Psi(\bullet, t) \stackrel{\text { def }}{=} \lim _{h \rightarrow 0} \frac{\Psi(\bullet, t+h)-\Psi(\bullet, t)}{h},
$$

provided the limit exists in $\mathbf{R}^{2} \otimes \mathscr{D}^{\prime}\left(\mathbf{R}_{x}\right)$.

To complete the definition of a distributional Lax pair we examine the conditions on the right hands sides of (3.1) needed to ensure that these are well defined distributions. In this letter we restrict our attention to the peakon ansatz (2.6). Away from the singular support of $m$, that is, away from the points $\left\{x_{1}, x_{2}, \cdots, x_{n}\right\}$, the distributional Lax pair will be smooth and given by (3.1). On 
the singular support the multipliers of $m$ are not continuous functions, because neither is $\Psi$ as a piecewise smooth function, and nor is $Q=u^{2}-u_{x}^{2}$, being a piecewise constant function (see the proof of Theorem 2.1), both with jumps on the singular support of $m$. Thus none of the multiplications $\Psi m, Q \Psi m$ is defined. To define them we will have to assign values to $\Psi$ and $Q$ at the points $\left\{x_{1}, x_{2}, \cdots, x_{n}\right\}$. We postulate that the values of $\Psi$ at these points are a linear combination of their respective left hand and right hand limits. More precisely

Definition 3.2. An invariant regularization of the Lax pair (3.1) valid for the peakon ansatz (2.6) is given by specifying the values of $\alpha, \beta \in \mathbf{R}$, subsequently setting

$$
\Psi\left(x_{k}, t\right)=\alpha[\Psi]\left(x_{k}, t\right)+\beta\langle\Psi\rangle\left(x_{k}, t\right),
$$

and assigning some values $Q\left(x_{k}, t\right)$ to $Q(x, t)=u^{2}-u_{x}^{2}$ at the points $\left\{x_{1}, x_{2}, \cdots, x_{n}\right\}$. Then

$$
\begin{array}{ll}
\Psi(\bullet, t) \delta_{x_{k}} \stackrel{\text { def }}{=} \Psi\left(x_{k}, t\right) \delta_{x_{k}}, & 1 \leq k \leq n, \\
Q(\bullet, t) \delta_{x_{k}} \stackrel{\text { def }}{=} Q\left(x_{k}, t\right) \delta_{x_{k}}, & 1 \leq k \leq n .
\end{array}
$$

Now the distributional Lax pair

$$
D_{x} \Psi=\frac{1}{2} U \Psi, \quad D_{t} \Psi=\frac{1}{2} V \Psi, \quad \Psi=\left[\begin{array}{l}
\Psi_{1} \\
\Psi_{2}
\end{array}\right],
$$

is well defined and we can ask meaningfully the question of compatibility, remembering that by Definition $3.1 D_{t}$ and $D_{x}$ indeed commute in action on $\Psi$. We have

Theorem 3.1. Let $m$ be the discrete measure associated to $u$ defined by (2.6). Given an invariant regularization in the sense of Definition 3.2 the distributional Lax pair (3.2) is compatible, i.e. $D_{t} D_{x} \Psi=D_{x} D_{t} \Psi$, if and only if the following conditions hold:

$$
\begin{aligned}
\beta=1, & \alpha^{2}=\frac{1}{4}, & Q\left(x_{k}\right) & =\langle Q\rangle\left(x_{k}\right), \\
\dot{m}_{k} & =0, & \dot{x}_{k} & =Q\left(x_{k}\right)
\end{aligned}
$$

We note that (3.3b) is precisely (2.12) whereas the choice of $\beta=1, \alpha=\frac{1}{2}$ and $\alpha=-\frac{1}{2}$ amounts to the choice of right limits, respectively left limits, in the definition of an invariant regularization 3.2 .

For a proof of Theorem 3.1 as well as a discussion of the origin of the concept of an invariant regularization we refer the reader to our longer paper [7] in which we give a complete solution to the integrable peakon problem (2.13); the solution is obtained by analyzing associated boundary value problems for the distributional Lax pairs (3.2) with $\beta=1, \alpha= \pm \frac{1}{2}$.

\section{Acknowledgements}

The first author was supported in part by National Research Science and Engineering Council of Canada (NSERC), the Department of Mathematics and Statistics of the University of Saskatchewan, the Pacific Institute of the Mathematical Sciences (PIMS) through the PIMS postdoctoral fellowship, and by LSEC, Institute of Computational Mathematics and Scientific Engineering Computing, AMSS, Chinese Academy of Sciences. J.S. was supported in part by NSERC \#163953. 


\section{References}

[1] R. Beals, D. H. Sattinger, and J. Szmigielski. Multi-peakons and a theorem of Stieltjes. Inverse Problems, 15(1):L1-L4, 1999.

[2] R. Beals, D. H. Sattinger, and J. Szmigielski. Multipeakons and the classical moment problem. Advances in Mathematics, 154:229-257, 2000.

[3] R. Beals, D. H. Sattinger, and J. Szmigielski. Inverse scattering solutions of the Hunter-Saxton equation. Appl. Anal., 78(3-4):255-269, 2001.

[4] P. M. Bies, P. Górka, and E. G. Reyes. The dual modified Korteweg-de Vries-Fokas-Qiao equation: geometry and local analysis. J. Math. Phys., 53(7):073710, 19, 2012.

[5] A. Bressan and A. Constantin. Global conservative solutions of the Camassa-Holm equation. Arch. Ration. Mech. Anal., 183(2):215-239, 2007.

[6] R. Camassa and D. D. Holm. An integrable shallow water equation with peaked solitons. Phys. Rev. Lett., 71(11):1661-1664, 1993.

[7] X.-K. Chang and J. Szmigielski. Lax integrability and the peakon problem for the modified CamassaHolm equation. to appear.

[8] M. Chen, S. Liu, and Y. Zhang. A two-component generalization of the Camassa-Holm equation and its solutions. Lett. Math. Phys., 75(1):1-15, 2006.

[9] A. Constantin. The trajectories of particles in Stokes waves. Invent. Math., 166(3):523-535, 2006.

[10] A. Constantin. Particle trajectories in extreme Stokes waves. IMA J. Appl. Math., 77(3):293-307, 2012.

[11] A. Constantin and J. Escher. Global weak solutions for a shallow water equation. Indiana Univ. Math. J., 47(4):1527-1545, 1998.

[12] A. Constantin and J. Escher. Wave breaking for nonlinear nonlocal shallow water equations. Acta Math., 181(2):229-243, 1998.

[13] A. Constantin and D. Lannes. The hydrodynamical relevance of the Camassa-Holm and DegasperisProcesi equations. Arch. Ration. Mech. Anal., 192(1):165-186, 2009.

[14] A. Constantin and W. A. Strauss. Stability of peakons. Comm. Pure Appl. Math., 53(5):603-610, 2000.

[15] A. Degasperis, D. Holm, and A. Hone. A new integrable equation with peakon solutions. Theor. Math. Phys., 133(2):1463-1474, 2002.

[16] A. Degasperis, D. D. Holm, and A. N. W. Hone. Integrable and non-integrable equations with peakons. In M. J. Ablowitz, M. Boiti, F. Pempinelli, and B. Prinari, editors, Nonlinear Physics: Theory and Experiment (Gallipoli, 2002), volume II, pages 37-43. World Scientific, 2003. Preprint nlin.SI/0209008.

[17] A. Degasperis and M. Procesi. Asymptotic integrability. volume 7, pages 23-37. (Rome, 1998) (A. Degasperis and G. Gaeta, eds.),World Scientific, New Jersey, 1999.

[18] A. Fokas. The Korteweg-de Vries equation and beyond. Acta Appl. Math., 39(1-3):295-305, 1995.

[19] A. S. Fokas. On a class of physically important integrable equations. Phys. D, 87(1-4):145-150, 1995. The nonlinear Schrödinger equation (Chernogolovka, 1994).

[20] L. Fontanelli, P. Lorenzoni, and M. Pedroni. A three-component extension of the Camassa-Holm hierarchy. Lett. Math. Phys., 78(2):125-137, 2006.

[21] B. Fuchssteiner. Some tricks from the symmetry-toolbox for nonlinear equations: generalizations of the Camassa-Holm equation. Physica D: Nonlinear Phenomena, 95(3):229-243, 1996.

[22] F. R. Gantmacher and M. G. Krein. Oscillation matrices and kernels and small vibrations of mechanical systems. AMS Chelsea Publishing, Providence, RI, revised edition, 2002. Translation based on the 1941 Russian original, edited and with a preface by Alex Eremenko.

[23] X. Geng and B. Xue. An extension of integrable peakon equations with cubic nonlinearity. Nonlinearity, 22:1847-1856, 2009.

[24] X. Geng and B. Xue. A three-component generalization of Camassa-Holm equation with N-peakon solutions. Adv. Math., 226(1):827-839, 2011.

[25] P. Górka and E. G. Reyes. The modified Camassa-Holm equation. Int. Math. Res. Not. IMRN, (12):2617-2649, 2011.

[26] G. Gui, Y. Liu, P. Olver, and C. Qu. Wave-breaking and peakons for a modified Camassa-Holm equation. Commun. Math. Phys., 319(3):731-759, 2013. 
[27] A. Himonas and D. Mantzavinos. The Cauchy problem for the Fokas-Olver-Rosenau-Qiao equation. Nonlinear Analysis: Theory, Methods \& Applications, 95:499-529, 2014.

[28] A. Himonas and D. Mantzavinos. The Cauchy problem for a 4-parameter family of equations with peakon traveling waves. Nonlinear Anal., 133:161-199, 2016.

[29] D. Holm and R. Ivanov. Two-component $\mathrm{CH}$ system: inverse scattering, peakons and geometry. Inverse Problems, 27(4):045013, 2011.

[30] A. Hone and J. Wang. Integrable peakon equations with cubic nonlinearity. J. Phys. A, 41(37):372002, 2008.

[31] A. N. W. Hone, H. Lundmark, and J. Szmigielski. Explicit multipeakon solutions of Novikov's cubically nonlinear integrable Camassa-Holm type equation. Dyn. Partial Differ. Equ., 6(3):253-289, 2009.

[32] J. Hunter and R. Saxton. Dynamics of director fields. SIAM J. Appl. Math, 51(6):1498-1521, 1991.

[33] J. K. Hunter and Y. X. Zheng. On a completely integrable nonlinear hyperbolic variational equation. Phys. D, 79(2-4):361-386, 1994.

[34] R. Ivanov. Extended Camassa-Holm hierarchy and conserved quantities. Z. Naturforschung A, 61:133138, 2006.

[35] J. Kang, X. Liu, P. J. Olver, and C. Qu. Liouville correspondence between the modified KdV hierarchy and its dual integrable hierarchy. J. Nonlinear Sci., 26(1):141-170, 2016.

[36] P. D. Lax. Integrals of nonlinear equations of evolution and solitary waves. Comm. Pure Appl. Math., 21:467-490, 1968.

[37] J. Lenells. A variational approach to the stability of periodic peakons. J. Nonlinear Math. Phys., 11(2):151-163, 2004.

[38] N. Li, Q. Liu, and Z. Popowicz. A four-component Camassa-Holm type hierarchy. Journal of Geometry and Physics, 85:29-39, 2014.

[39] Z. Lin and Y. Liu. Stability of peakons for the Degasperis-Procesi equation. Comm. Pure Appl. Math., 62(1):125-146, 2009.

[40] X. Liu, Y. Liu, and C. Qu. Orbital stability of the train of peakons for an integrable modified CamassaHolm equation. Adv. Math., 255:1-37, 2014.

[41] H. Lundmark and J. Szmigielski. Degasperis-Procesi peakons and the discrete cubic string. IMRP Int. Math. Res. Pap., (2):53-116, 2005.

[42] H. Lundmark and J. Szmigielski. An inverse spectral problem related to the Geng-Xue two-component peakon equation. Memoirs of Amer. Math. Soc., 244(1155), 2016.

[43] A. V. Mikhailov and V. S. Novikov. Perturbative symmetry approach. J. Phys. A, 35(22):4775-4790, 2002.

[44] V. Novikov. Generalisations of the Camassa-Holm equation. J. Phys. A, 41(37):372002, 2008.

[45] P. Olver and P. Rosenau. Tri-Hamiltonian duality between solitons and solitary-wave solutions having compact support. Phys. Rev. E, 53(2):1900, 1996.

[46] Z. Qiao. A new integrable equation with cuspons and W/M-shape-peaks solitons. J. Math. Phys., 47(11):112701-112900, 2006.

[47] Z. Qiao. New integrable hierarchy, its parametric solutions, cuspons, one-peak solitons, and M/W-shape peak solitons. J. Math. Phys., 48(8):082701, 2007.

[48] Z. Qiao. M-shape peakons, dehisced solitons, cuspons and new 1-peak solitons for the DegasperisProcesi equation. Chaos, Solitons \& Fractals, 37(2):501-507, 2008.

[49] Z. Qiao, B. Xia, and J. Li. Integrable system with peakon, weak kink, and kink-peakon interactional solutions. arXiv:1205.2028, 2012.

[50] C. Qu, X. Liu, and Y. Liu. Stability of peakons for an integrable modified Camassa-Holm equation with cubic nonlinearity. Comm. Math. Phys., 322(3):967-997, 2013.

[51] J. Song, C. Qu, and Z. Qiao. A new integrable two-component system with cubic nonlinearity. J. Math. Phys., 52(1):013503, 2011. 